\title{
Simulation and Calculation of Residual Stresses in Mining Machines Components
}

\author{
Valeriy Blumenstein ${ }^{*}, 1$, Maksim Mahalov ${ }^{1}$, and Oleg Ostanin ${ }^{1}$ \\ ${ }^{1}$ T.F. Gorbachev Kuzbass State Technical University, Vesennyaya street 28, Kemerovo, 650000, \\ Russian Federation
}

\begin{abstract}
Technologies of surface plastic deformation (SPD) provide a hardening effect and create compressive residual stresses in the surface layer of mining machine parts. This leads to a significant increase in the limit of endurance and fatigue cyclic life, increases the life in fretting conditions, improves the quality of assembly joints fit, etc. Very complex are both the physical nature of the occurrence and the estimation of residual stresses, since the machining processes are related to complex nonmonotonic types of loading. The study shows the relevance of the development of computational methods for determining residual stresses in the processes of hardening treatment of critical surfaces of mining machine parts. At the same time, the design and development of experimental nondestructive express methods based on the determination of various physical parameters (acoustic, magnetic noise, coercive force, etc.) are relevant and promising. A model of the process is developed and numerical calculations of the residual stresses arising during the SPD treatment are carried out. The results of the calculations are in good agreement with the experimental data.
\end{abstract}

\section{Introduction}

Hardening during surface plastic deformation (SPD) treatment significantly increases fatigue resistance, fatigue cyclic life and contributes to a greater life before the appearance of a fatigue crack [1]. For steels with a low carbon content $(0.2 \%)$, the effect of increasing the fatigue strength is due to an increase in surface hardness (hardening), whereas for solid steels, it is explained to a great extent by the emergence of compressive residual stresses that inhibit the appearance and development of small cracks.

I. Altenberger notes that the SPD treatment is used in many fields of engineering, both to improve the surface quality, and to improve the strength of metal parts of machines in fatigue conditions, fretting loads, etc. [1]. They include: rotationally symmetric components of cars (axles, shafts and elements of steering joints), shafts of reducers for agricultural, road and mining machines, military aircraft wheel disks, high-loaded screws and bolts, as well as turbine disks, compressor fan blades, etc. Shot Peening (SP) and Deep Cold Rolling (DCR) are used to create Compressive Residual Stress (CRS) on the surface and over the depth of the hardened layer [2]. In this case, Vibro-Peening (VP) technology can be used as

\footnotetext{
*Corresponding author: blumenstein@rambler.ru
} 
an alternative to SP to create hardening and residual compressive stresses with significantly less roughness. Good results in the processing of titanium alloy Ti6Al4V were achieved by combining technologies (SP), (DCR) and (VP) [3]. Lim et al. demonstrated that the net movement of material in the surface region led directly to larger plastic strains and hence more compressive residual stress, through the finite element process modelling of a full DCR treatment [4].

Abrasive waterjet (AWJ) of Ti6Al4V titanium alloy and ASTM A228 steel was carried out on the surfaces elastically prestressed at the values ranging from 0 to $75 \%$ of the substrate yield strength. The surface residual stress ranged from 680 to $1487 \mathrm{MPa}$ for Ti6Al4V and 720 to $1554 \mathrm{MPa}$ for ASTM A228 steel; the depth ranged from 265 to 370 $\mu \mathrm{m}$ for Ti6A14V and 550 to $680 \mu \mathrm{m}$ for ASTM A228 steel. Results showed that elastic prestress may be used to increase the surface residual stress in AWJ peened components by up to $100 \%$ [5].

The effect of hardening and compressive residual stresses was studied for various parts of various materials. For example, in the study of W.J. Qin, C. Dong and X. Li, the evaluation was made, and the effect of increasing the fatigue strength of the crankshaft, taking into account the RSs was shown [6].

Most researchers agree that it is important to measure, evaluate, predict and control their magnitude and distribution of residual stresses. Residual stresses are investigated by experimental methods, including tensometry [2], evaluation of deformations in the relaxation of residual stresses [5], by layer-by-layer chemical removal of hardened material layers with an estimation of the emerging deformation [5], X-ray diffraction [7], microindentation [8] and others.

It was shown in the study [9] that the field of residual stresses is the energy field of deformation. The distribution of residual stresses, including the numerical value and the existing range, correlates with the amount of energy. On this basis, an integral of the strain energy density is proposed. Under experimental conditions, it is established that frictional forces and thermal effects play a major role in determining the final distribution of residual stresses.

The most adapted for solving the problems of estimating residual stresses after hardening in machining processes and their effect on fatigue strength is the finite element method (FEM).

In the already mentioned article [6], FEM is used to simulate the behavior of the crankshaft under fatigue loading with allowance for residual stresses. Xiaoming Huang, Jie Sun and Jianfeng Li performed the FEM simulation and experimental study of residual stresses. The results of FEM simulation and experimental studies showed high convergence with the experimental data [10]. The results of numerical modeling of residual stresses in cutting processes are presented in the studies [11-14], taking into account friction, temperatures, lubricants, etc. when processing different materials with different tools.

T. Altan conducted a study of the mechanics of the SPD process, having created 2D and 3D FEM models of roller rolling [15]. The simulation results, including surface deformations and residual stresses, showed high convergence with the experimental data obtained from literature sources.

\section{Theoretical studies}

The authors note that at least two problems remain relevant in this area of knowledge.

First, the complexity and rather high labour intensity of the experimental determination of residual stresses based on the destruction of the part and the layer-by-layer removal of the surface layer metal and the measurement of the residual deformations of the part with subsequent calculation of RS values. X-ray methods make it possible to detect RS mainly in 
thin surface layers, estimated at $10-20 \mu \mathrm{m}$; while it is not possible to determine the RS distribution over the depth of the hardened surface layer. At the same time, design and development of experimental express methods based on methods of nondestructive testing (NDT) and determination of various physical parameters (acoustic, magnetic noise, coercive force, etc.) are urgent and promising.

Secondly, currently known analytical models do not allow us to calculate residual stresses with satisfactory accuracy for complex types of nonmonotonic loading types, which include most of the machining methods.

Within the framework of the developed theory of the mechanics of technological inheritance, a model of a hardened body was proposed, implemented for the method of surface plastic deformation [16]. Theoretical studies were carried out using FEM in a flat setting, according to which the process was simulated in the plane of feed, passing through the axis of rotation of the workpiece being processed; accepted material model - elasticplastic with hardening [17-20].

According to the unloading theorem, the residual stress tensor is the difference in stresses arising in a real elastoplastic body when a load $\left[T \sigma_{\text {def }}\right]$ is applied and the stresses that would appear in a perfectly elastic body under identical loading $\left[T \sigma_{\text {elast }}\right]$ summed with the elastic unloading stresses of the unfastening of the workpiece $\left[T \sigma_{\operatorname{det}}\right]$ and the elastic thermal stresses of the unloading $\left[T \sigma_{t^{0}}\right]$ :

$$
\left[T \sigma_{\text {res }}\right]=\left[T \sigma_{\text {def }}\right]-\left[T \sigma_{\text {elast }}\right]+\left[T \sigma_{\text {det }}\right]+\left[T \sigma_{t^{0}}\right]
$$

To solve this problem, a finite-element model of the formation of the mechanical state was developed for a hardening treatment of a surface layer (SL) of a SPD metal by a roller tool, taking into account the transformation of the metal properties in the deformation zone (DZ). When creating the model, the following initial / boundary conditions and assumptions were accepted: the simulated material (steel 45) was assumed to be isotropic; the arising deformations had only a mechanical nature due to the small heating under the considered loading; coefficient of friction in the contact pair $\eta=0,21$; bilinear approximation of the flow curve was used, taking into account the hardening of the metal (Fig. 1). To simplify the calculations, due to the relatively low magnitude of the resulting error, the Bauschinger effect was not taken into account; the stress tensor of elastic unloading during the detachment of the workpiece was taken as zero; the model of the part had large enough geometric dimensions in comparison with the emerging zone of deformation

The mode of SPD treatment: profile radius of the roller $R_{p r}=5 \mathrm{~mm}$; feed S $=0.1 \mathrm{~mm} /$ rev; roller tension $h_{r}=0.05 \mathrm{~mm}$.

Taking into account the assumptions, the tensor of residual stresses in the coordinate system associated with the shape of the part or a structural element was represented in the following form:

$$
\left[T \sigma_{\text {res }}\right]_{x y z}=\left(\begin{array}{ccc}
\sigma_{x}^{\text {def }}-\sigma_{x}^{\text {elast }} & \sigma_{x y}^{\text {def }}-\sigma_{x y}^{\text {elast }} & 0 \\
\sigma_{x y}^{\text {def }}-\sigma_{x y}^{\text {elast }} & \sigma_{y}^{\text {def }}-\sigma_{y}^{\text {elast }} & 0 \\
0 & 0 & \frac{\left(\sigma_{x}^{\text {def }}+\sigma_{y}^{\text {def }}\right)-\left(\sigma_{x}^{\text {elast }}+\sigma_{y}^{\text {elast }}\right)}{2}
\end{array}\right)
$$


where: $\sigma_{x}^{\text {def }}, \sigma_{y}^{\text {def }} \sigma_{x y}^{\text {def }}$ - components of the stress tensor appearing in a real elastoplastic body when a load is applied; $\sigma_{x}^{\text {elast }}, \sigma_{y}^{\text {elast }}, \sigma_{x y}^{\text {elast }}$ - components of the stress tensor, which would arise in an ideal-elastic body under identical loading.

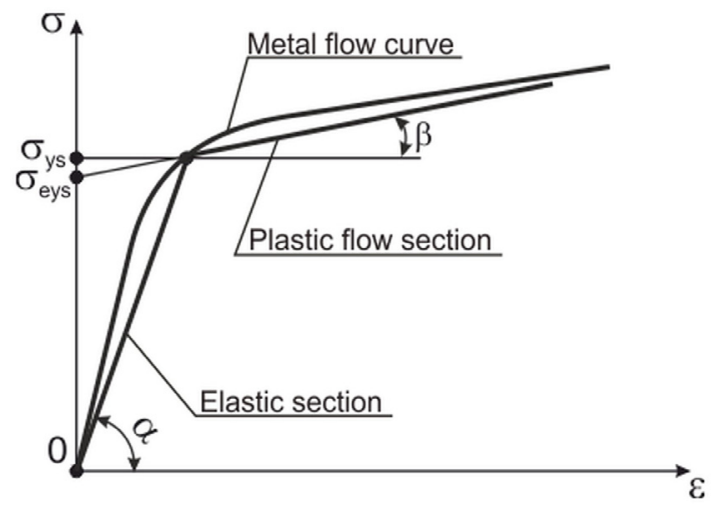

Fig. 1. Bilinear approximation of the flow curve.

As a machined surface, a fragment of the plane of the main deformations of a cylindrical piece with a length $\mathrm{L}=50 \mathrm{~mm}$ and height $\mathrm{h}=20 \mathrm{~mm}$ was simulated (Fig. 2), the lower and lateral boundaries of which were rigidly fixed on both axes. The dimensions of the deformation arising during the processing of the deformation zone were much less than the modeled fragment, so the edge effects that arise in this arrangement produce a very insignificant error in the simulation results (Fig. 3). For the convenience of further calculations, a grid of square elements $0.5 \mathrm{~mm}$ in size was generated on the model of the workpiece. The indenter was modeled as an absolutely rigid body and was a circle modeling a roller of a given profile radius.

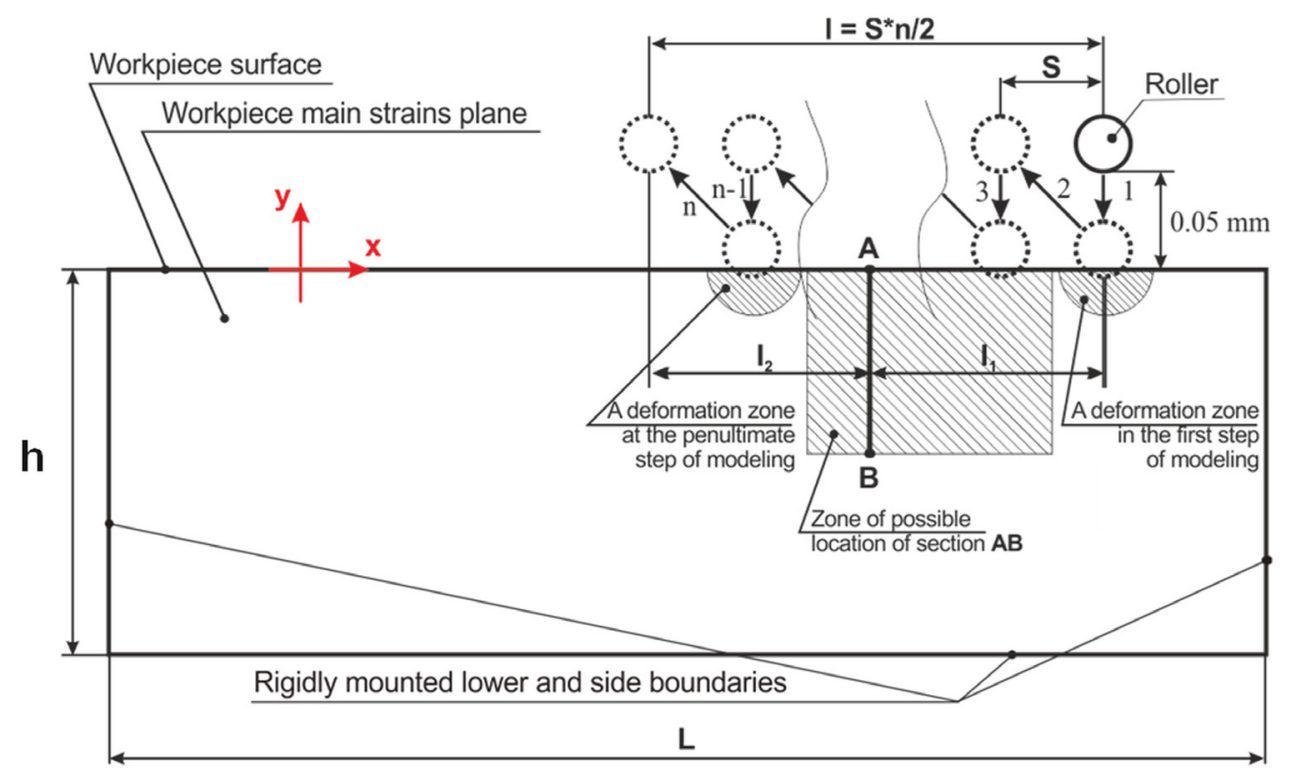

Fig. 2. Statement of problem in SPD treatment simulation. 


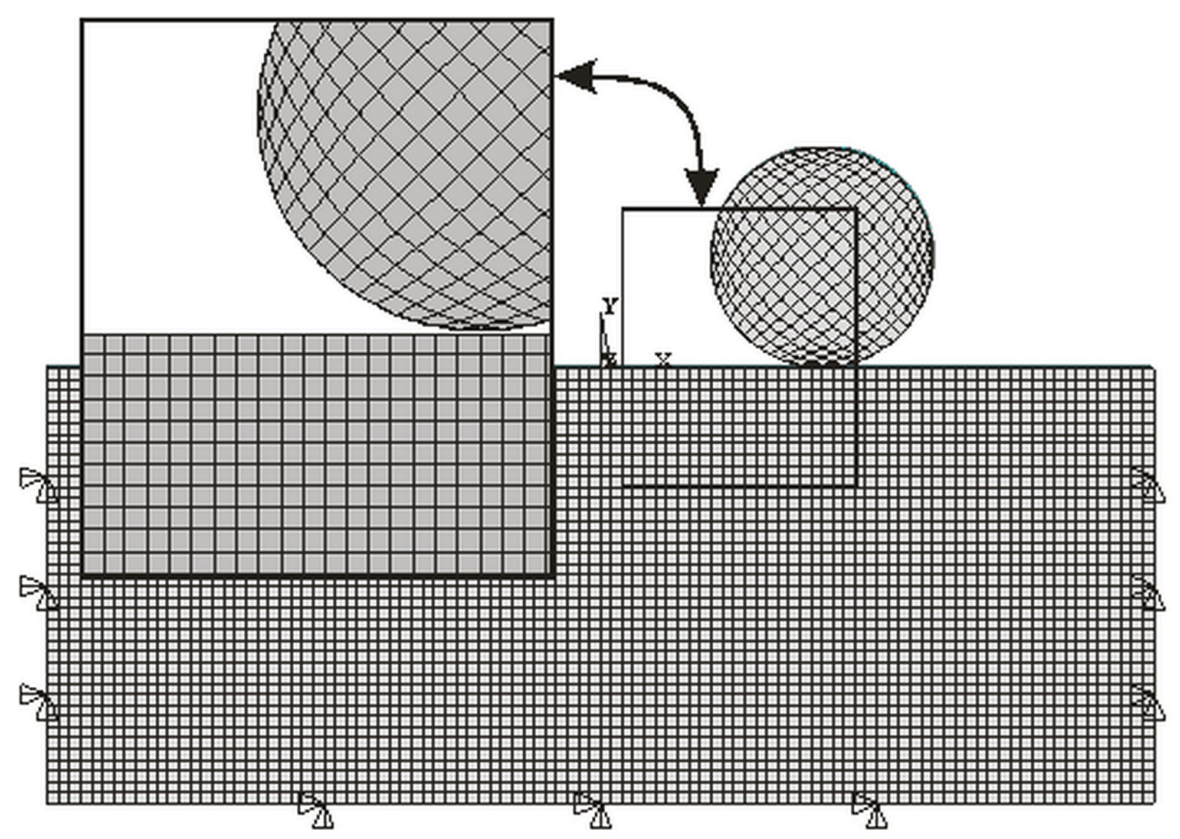

Fig. 3. Breaking up the model into finite elements.

In the initial position, the indenter was relative to the surface with a certain gap. At the first and any subsequent odd simulation step, the load was applied - the displacement of the indenter in the direction of the surface by a certain amount, assuming the introduction and creation of a predetermined interference simulation. At the second and any subsequent even step of the simulation, unloading was performed - the withdrawal of the indenter from the surface to the initial distance, with its simultaneous displacement along the surface by the amount of feed. In total, 300 steps were simulated - 150 loading steps and 150 unloading steps. In this case, taking into account the accepted feed rate, the length of the treated surface in the presented model was $l=S^{*} n / 2=15 \mathrm{~mm}$. The main idea of the simulation was that some section $A B$ of the modeled fragment is located in such a way that at the 1 st step of loading it does not yet enter the DZ space. At the same time, at the penultimate step of loading, this section has already left the DZ space. In the model under consideration, the distance $\boldsymbol{l}_{\boldsymbol{l}}$ in the feeding direction from the starting position of the indenter to the cross section $A B$ was $5 \mathrm{~mm}$, the distance $l_{2}$ in the feeding direction from the cross section $A B$ to the final position of the indenter was $10 \mathrm{~mm}$

In the plane of the main deformations (the feed plane, Fig. 1), the movement of the DZ in the feeding direction is of a discrete nature: a new DZ arises with a shift relative to the previous one by the amount of feed.

Thus, in the course of realization of all simulation steps, this cross-section passes through the DZ space and is the cross-section over the depth of the treated surface layer SL in which the accumulation of deformation, a partial depletion of the plasticity reserve, and the formation of the RS tensor took place taking into account the changing properties of the SL.

\section{Results and discussion}

After solving the model in the selected cross section $A B$, the following values were recorded for each simulation step: the node coordinates, the components of the nodes 
displacement vector, the tensor components of the elastic, plastic and total elastic-plastic deformation, and also the stress tensor components: stresses under load for odd modeling steps (loading steps), intermediate residual stresses for even simulation steps (unloading steps), and the total residual stresses for the last even step of the simulation, when the reviewed cross-section left the space of the deformation zone.

Let us consider in more detail the numerical values of the stress state components at the last two modeling steps: step 299 (loading) and step 300 (unloading) (Fig. 4). The axial component $\sigma_{\mathrm{x}}$ varies under load in the range $1150 \ldots 400 \mathrm{MPa}$, and in the unloading cycle (axial RS) in the range $-680 \ldots 480 \mathrm{MPa}$, while in both cases a layer with compressive residual stresses up to $-680 \mathrm{MPa}$ is observed throughout the treated surface. The radial component $\sigma_{\mathrm{y}}$ under load also varies in a significant range $-1920 \ldots 100 \mathrm{MPa}$, while in the unloading cycle there are small compressive and tensile stresses of $-170 \ldots 150$ $\mathrm{MPa}$ observed. As a result, tensile RSs of small size (up to $100 \mathrm{MPa}$ ) are formed on the treated surface. In accordance with the accepted statement of modeling, the circumferential component is a half-sum of the axial and the radial one, reflecting accordingly the tendency of their joint distribution. The shear component $\sigma_{x y}$ in the simulated plane at the loading stage is characterized by a range of values $-300 \ldots 460$ $M P a$, while in the forward area of the deformation zone there are negative values, in the rear area the values are positive. At the stage of unloading, the shear stresses have approximately equal values of both signs $(-180 \ldots 200 \mathrm{MPa})$, which on average are 2 times lower than the values in the loading cycle. At the same time, no significant values of this component over the depth of the treated surface layer are observed. The average normal stress varies in the ranges $-1460 \ldots 30 \mathrm{MPa}$ and $-430 \ldots 70 \mathrm{MPa}$, respectively, in the penultimate and final modeling steps. The presented picture allows us to conclude that, in general, most of the space of the deformation zone, like the surface layer of the part obtained as a result of treatment, is mainly in the state of compression.

The results of modeling and calculations by this method showed that after SPD treatment, the largest values of the compressive residual stresses (up to -600 MPa) are characteristic for the axial component $\sigma_{x}$, while the maximum values for the treatment mode under consideration are located on the surface of the part. The depth of propagation of compressing RSs is approximately $3 \mathrm{~mm}$ with a general depth of propagation of significant stresses of about $7 \mathrm{~mm}$ (Fig. 5).

The tangential (circumferential) component $\sigma_{z}$ has values of compressive stresses comparable to the axial component (up to $-520 \mathrm{MPa}$ ). The maximum values also occur on the surface of the part. The radial component $\sigma_{y}$ is characterized by much smaller values than $\sigma_{x}$ values at the same depths of propagation. On the surface and in the subsurface layer, $\sigma_{y}$ takes predominantly negative values, reaching $-50 \mathrm{MPa}$. It is also characterized by smaller drops of values, both in the area of the tensile and in the area of compressive values.

The values obtained correspond to the experimental data, according to which the value of the axial residual stresses at SPD reaches $-800 \mathrm{MPa}$, the circumferential stresses -400 $\mathrm{MPa}$; the radial stresses are close to zero. The small values of $\sigma_{y}$ which are characteristic for the RS tensor at SPD, are not a significant drawback due to the minor effect of this component on the cyclic life of the part. The components of the RS tensor are characterized by a considerable (up to $\mathrm{h}=4 \mathrm{~mm}$ ) depth of propagation of the compressive stresses, as well as a significant (up to $\mathrm{h}=7 \mathrm{~mm}$ ) propagation depth. 

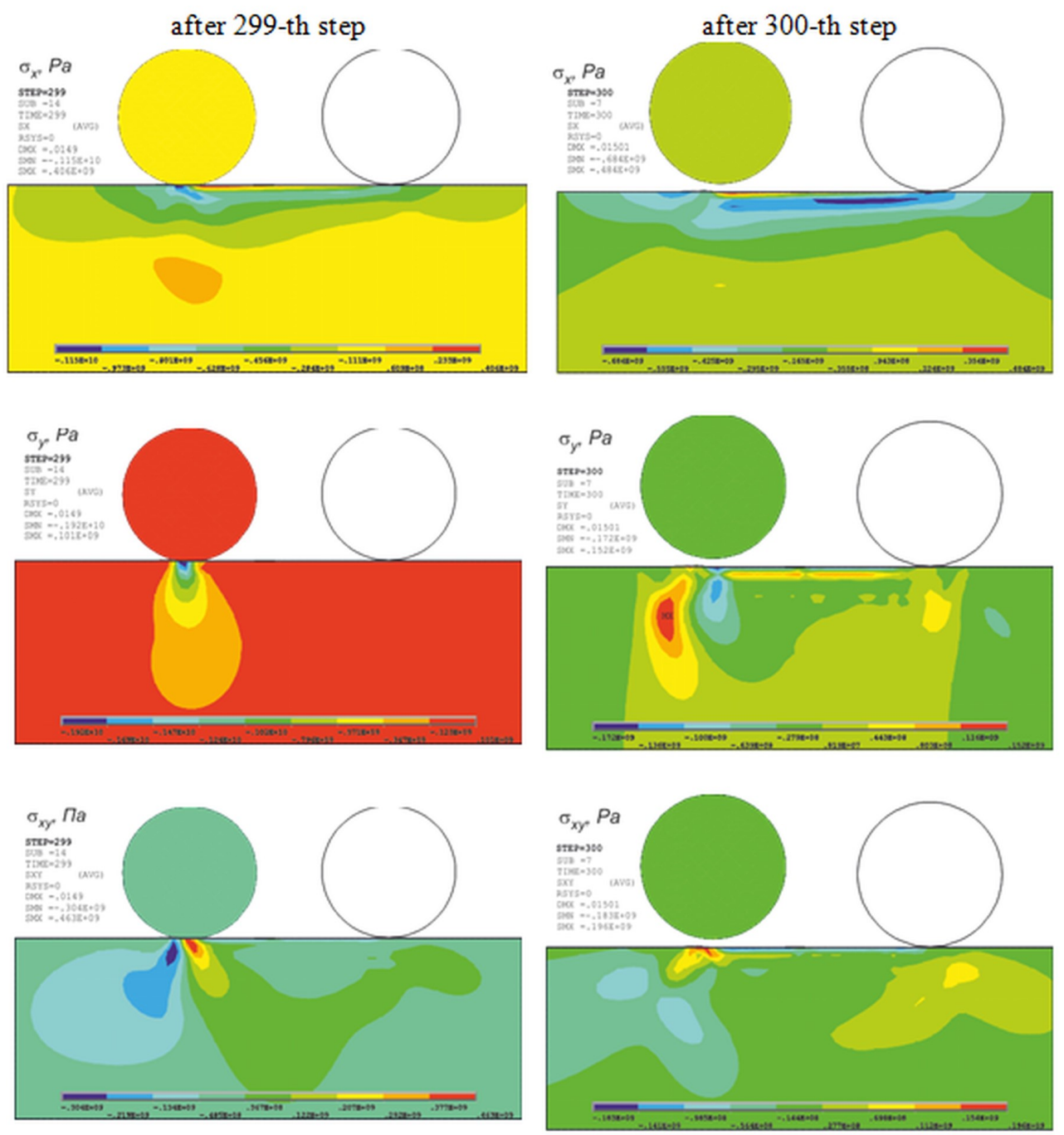

Fig. 4. The residual stress tensor components after the 299-th and the 300-th simulation step

\section{Conclusions}

The urgency of developing computational methods for determining residual stresses in the processes of hardening treatment is shown. The importance of using surface plastic deformation (SPD) technologies providing a hardening effect and creating compressive residual stresses in the surface layer of machine parts is highlighted.

The FEM-model is developed, which is based on the idea of the sequential loading and unloading of the deformation zone that occurs in the contact zone of the deforming roller with the surface layer of the part.

The analysis and numerical calculations of the residual stresses arising during the SPD treatment are performed. The results of the calculations are in good agreement with the experimental data. 


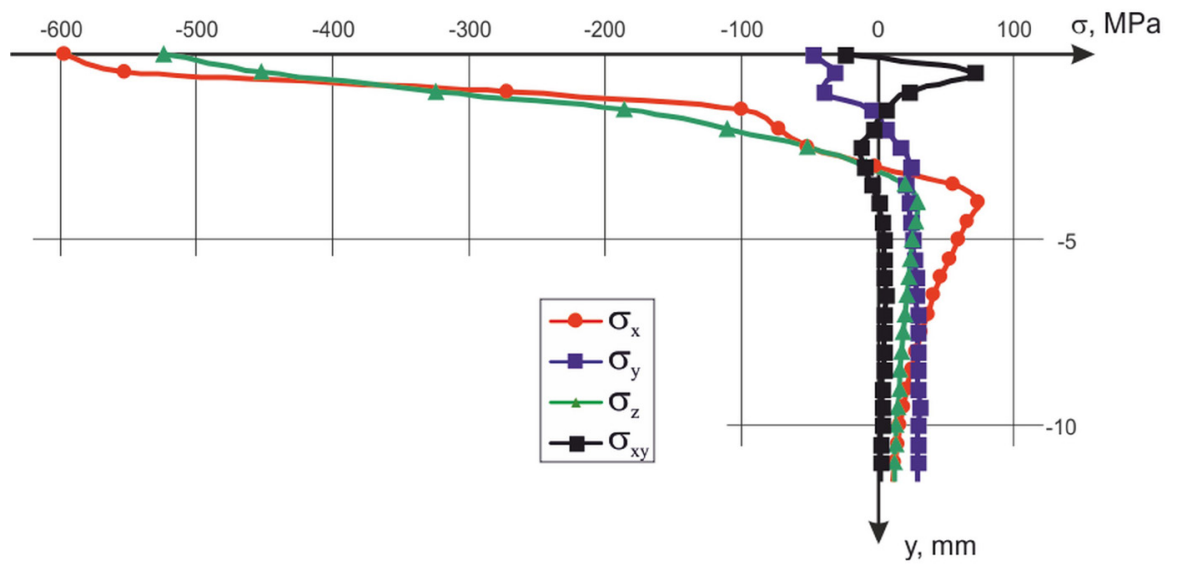

Fig. 5. The residual stress tensor components (after the 300-th simulation step) in the surface layer depth.

\section{References}

1. I. Altenberger, 9th International Conference on Shot Peening (ICSP9), 144 ( 2005)

2. A. Gopinath, A. Lim, B. Nagarajan, C. C. Wong, R. Maiti, S. Castagne, IOP Conf. Series: Materials Science and Engineering, 157, 012013 (2016)

3. G. H. Majzoobi, K. Azadikhah, J. Nemati, Mater. Sci. Eng. A, 516:1-2, 235 (2009) doi: 10.1016/j.msea.2009.03.020

4. A. Lim, S. Castagne, C. C. Wong, Journal of Manufacturing Science and Engineering, 138:11 (2016) doi: 10.1115/1.4033524

5. B. Sadasivam, A. Hizal, D. Arola ASME International Mechanical Engineering Congress and Exposition, IMECE 2007, 43473 (2007)

6. W. J. Qin, C. Dong, X. Li, JMEPEG, 25, 938 (2016) doi: 10.1007/s11665-016-1890-1

7. S. Saini, I. S. Ahuja, V. S. Sharma, Int. J. Adv. Manuf. Technol., 65, 667 (2013) doi:10.1007/s00170-012-4206-0

8. A. Yonezu, R. Kusano, T. Hiyoshi, X. Chen, JMEPEG, 24:362 (2015) doi: $10.1007 / \mathrm{s} 11665-014-1280-5$

9. Y. Ma, P. Feng, J, Zhang, Z. Wu, D. Yu, Int. J. Adv. Manuf. Technol. 81, 1023 (2015) doi: 10.1007/s00170-015-7278-9

10. X. Huang. J. Sun. J. Li, Int. J. Adv. Manuf. Technol., 77:5-8, 1035 (2015)

11. J.-C. Su, K. A. Young, K. Ma, S. Srivatsa, J. B. Morehouse, S. Y. Liang, Int. J. Adv. Manuf. Technol., 65, 717 (2013) doi: 10.1007/s00170-012-4211-3

12. X. Ji, X. Zhang, S. Y. Liang, Int. J. Adv. Manuf. Technol., 70, 2159 (2014) doi:10.1007/s00170-013-5439-2

13. J. J. Martell, C. R. Liu, J. Shi, Int. J. Adv. Manuf. Technol., 74, 1381 (2014) doi: 10.1007/s00170-014-6089-8

14. J. Chen, Q. Fang, L. Zhang, Int. J. Adv. Manuf. Technol., 75, 615 (2014) doi: 10.1007/s00170-014-6128-5

15. Y. C. Yen, P. Sartkulvanich, T. Altan, CIRP Annals, 54: 1, 237 (2005) doi:10.1016/S0007-8506(07)60092-4

16. V. Yu. Blumenstein, International journal for science and innovations for the industry. 
Innovations in discrete productions, III:2, 18 (2015)

17. M. S. Mahalov, V. Yu. Blumenstein, IOP Conf. Series, Materials Science and Engineering, 253:1, 012009 (2017)

18. V. Yu. Blumenstein, M. S. Mahalov, A.G. Shirokolobova, IOP Conf. Series:: Materials Science and Engineering, 253:1, 012017 (2017)

19. M. S. Mahalov, V. Yu. Blumenstein, Russ. Engin. Res., 36:7, 520 (2016) doi: 10.3103/S1068798X16070157

20. M. S. Mahalov, V. Yu. Blumenstein, IOP Conf. Series. Materials Science and Engineering, 126:1, 012004 (2016) 